\title{
Measurement and correction of the slope angle of flat surfaces digitized by a conoscopic holography system
}

\author{
Gonzalo Valiño*, Pablo Zapico, Pedro Fernández, J. Carlos Rico, Sabino Mateos, \\ David Blanco
}

Department of Construction and Manufacturing Engineering, University of Oviedo, Campus de Gijón, 33203 Gijón, Spain

\section{A R T I C L E I N F O}

\section{Article history:}

Received 13 October 2015

Received in revised form 14 April 2016

Accepted 11 June 2016

Available online 14 June 2016

\section{Keywords:}

Conoscopic holography

Non-contact inspection

Slope angle

CMM

\begin{abstract}
A B S T R A C T
Conoscopic Holography $(\mathrm{CH})$ is a non-contact interferometric technique used in surface digitizing. Similarly to other laser techniques it is influenced by the optical behaviour of the part surface and the conditions during scanning (geometry, strategy, etc.). In this work, a $\mathrm{CH}$ system and a touch probe (TP) integrated in a CMM were used to analyse the $\mathrm{CH}$ behaviour for measuring the slope angle of flat surfaces in inspection tasks. For this purpose, several digitizing tests were performed with both sensors on a square specimen under different slope angles. The tests were performed for two different orientations of the laser spot as well as at three different positions within the sensor depth of field (DOF). The specimen angles determined by each sensor were compared to each other and the difference (slope deviation) was used as an indicator of the $\mathrm{CH}$ behaviour. Considering the results, some recommendations were provided for digitizing sloped flat surfaces with the $\mathrm{CH}$ sensor and it was also developed a model to predict and compensate the measurement deviations of this sensor with regard to the TP.
\end{abstract}

(c) 2016 Elsevier Inc. All rights reserved.

\section{Introduction}

Industrial use of commercial scanners like non-contact digitizing systems has grown significantly in recent years with a wide range of applications that go from dimensional metrology to reverse engineering [1-3]. Apart from avoiding contact with the object to be measured, the main advantages over contact systems are the ability to capture small geometries and complex shapes as well as the high rate for points acquisition. Additionally, the portability of non-contact systems offers the possibility to be installed on different equipment such as coordinate measuring machines (CMM), articulated arm coordinate measuring machines, machine tools or production systems, which certainly favours its industrial application.

Despite the above advantages, commercial non-contact scanners are usually less accurate than the traditional contact-type methods, since their accuracy depends strongly on the relative position and orientation of the sensor with regard to the digitized part, the configuration parameters of the sensor, the part geometry, the optical properties of material, the surface roughness, etc.

\footnotetext{
* Corresponding author.

E-mail address: gvr@uniovi.es (G. Valiño).
}

Currently, there exist numerous non-contact techniques for surface digitizing, such as those based on triangulation laser which are more deeply analysed and disseminated every day [4-11]. Some of them [4-6] examined the effect of scan distance and beam incident angle on the random and systematic errors of the scanner. In the case of Feng et al. [4] and Isheil et al. [6] also provided models for compensating the measurement errors.

Using a triangulation laser scanner installed in a CNC platform Vukašinović et al. [8] found that the intensity of the light reflected by a digitized surface decreased as the surface slope increased. The best measurement results were obtained for surfaces with a good diffuse reflection and nearest to the sensor. Considering these influence factors the authors provided a mathematical model to predict the measurement uncertainty for a laser-scanning process [9].

The study presented by Bin et al. [10] used a laser sensor mounted on a 5-axes machining centre to analyse the effects of the position and orientation on the laser measurement of free-form surfaces. To improve the measurement accuracy, a measurement strategy that considered the position and orientation of the sensor and a semi-quantitative error-compensation model based on geometrical optics was proposed. Manorathna et al. [11] presented a set of performance evaluation tests for a 3D laser scanner attached to a 6 -axes robot. The scanner was evaluated under operating conditions such as different surface reflectivity, view angle, surface roughness and stand-off distance. The best working range was 